\title{
CDISC SDTM Pharmacokinetic Parameter Unit of Measure Terminology
}

National Cancer Institute

\section{Source}

National Cancer Institute. CDISC SDTM Pharmacokinetic Parameter Unit of Measure

Terminology. NCI Thesaurus. Code C85494.

Terminology associated with the pharmacokinetic parameter unit of measure codelist of the Clinical Data Interchange Standards Consortium (CDISC) Study Data Tabulation Model (SDTM). 